\title{
Edge plasma density reconstruction for fast monoenergetic lithium beam probing
}

\author{
S. Sasaki and S. Takamura \\ Department of Electrical Engineering and Electronics, School of Engineering, Nagoya University, Nagoya \\ 464-01, Japan \\ M. Ueda, ${ }^{a)} H$. Iguchi, and J. Fujita \\ National Institute for Fusion Science, Nagoya 464-01, Japan \\ K. Kadota \\ Plasma Science Center, Nagoya University, Nagoya 464-01, Japan
}

(Received 14 April 1992; accepted for publication 7 April 1993)

\begin{abstract}
Two different electron density reconstruction methods for 8-keV neutral lithium beam probing have been developed for the Compact Helical System (CHS). Density dependences on emission and ionization processes are included by using effective rate coefficients obtained from the collisional radiative model. Since the two methods differ in the way the local beam density in the plasma is determined, the methods have different applicable electron densities. The beam attenuation is calculated by iteration from the electron density profile in method I. In method II, the beam remainder at the observation point $z$ is determined by integrating the $\mathrm{Li} \mathrm{I}$ emission intensity from $z$ toward the position of emission tail-off. At the emission tail-off, the fast lithium beam is completely attenuated. Selecting an appropriate method enables us to obtain edge electron density profile well inside the last closed flux surface for various ranges of plasma densities $\left(10^{12}-5 \times 10^{13} \mathrm{~cm}^{-3}\right)$. The electron density profiles reconstructed by these two different methods are in good agreement with each other and are consistent with results from ruby laser Thomson scattering.
\end{abstract}

\section{INTRODUCTION}

The determination of plasma parameters at the edge region is important in the recent fusion research from the viewpoints of the generation and screening of impurities, and the improvement of global plasma confinement. Neutral lithium $\left(\mathrm{Li}^{0}\right)$ beam probing is now considered as one of the most powerful techniques for measuring the edge plasma density without perturbation or contamination to the plasma. ${ }^{1}$ By the use of a 4-keV beam, the electron density profile was successfully obtained with a good spatial resolution $(\sim 0.8 \mathrm{~cm})$ from the emission intensity of the $\mathrm{Li}$ I resonance line in NBT. ${ }^{2}$ It is, however, difficult to apply this simple method to larger plasma devices like tokamaks and helical devices. This is because the beam attenuation becomes severe and complicated atomic processes involving plasma electrons, protons, and impurity ions cannot be neglected. Hence, density reconstruction considering beam transport in the plasma is necessary. More energetic $\mathrm{Li}^{0}$ beams have been used to get larger penetration for higher density plasmas in TEXTOR ${ }^{3}(20-30 \mathrm{keV})$ and $\operatorname{ASDEX}^{4}(40-100 \mathrm{keV})$. In these cases, however, neglecting the delay of the Li I emission due to the finite lifetime of the lithium-excited state makes the spatial resolution worse $(2-4 \mathrm{~cm})$. Moreover, the reconstruction procedure is more complicated since charge transfer to plasma protons and impurity ions becomes dominant for the attenuation of such high-energy beams.

A beam energy of $8 \mathrm{keV}$ is selected for the fast

\footnotetext{
*) Permanent address: Instituto Nacional de Pesquisas Espaciais, São José dos Campos, São Paulo, 12201, Brazil.
}

$\mathrm{Li}^{0}$-beam probe system on the Compact Helical System (CHS) $)^{5}$ because it offers an adequate spatial resolution $(\sim 1.3 \mathrm{~cm})$ and beam penetration. The beam penetration depth $l_{p}$ is expressed in terms of $\overline{n_{e}} \times l_{p}$, where $\overline{n_{e}}$ is the average electron density. The value of $\bar{n}_{e} \times l_{p}$ is evaluated as $2.6 \times 10^{14} \mathrm{~cm}^{-2}$ for an $8-\mathrm{keV}$ beam. In this article, we show that it is possible to measure the edge electron density profile for a wide range of plasma densities by using two different density reconstruction methods. In method I, the beam attenuation due to ionization is considered. This is a standard approach for including the beam attenuation in $\mathrm{Li}^{0}$-beam probing and in charge-exchange recombination spectroscopy. ${ }^{6}$ In CHS this method is used to measure relatively low-density plasmas $\left(\overline{n_{e}}<3 \times 10^{13} \mathrm{~cm}^{-3}\right)$. On the other hand, method II is applied to relatively high-density plasmas $\left(\bar{n}_{e}>3 \times 10^{13} \mathrm{~cm}^{-3}\right)$ where the injected fast $\mathrm{Li}^{0}$ beam is completely attenuated within the observation window. The local beam density at position $z$ is determined by integrating the Li $I$ emission intensity from $z$ to the emission tail-off. In other words, the beam remainder at position $z$ is measured. Method II has been applied to $\mathrm{Li}^{0}$-beam probing using thermal type $e^{7,8}$ and laser blow-off type $e^{9-12}$ beam sources, which have much smaller penetration depths $\left(\overline{n_{e}} \times l_{p}=10^{12}-10^{13} \mathrm{~cm}^{-2}\right)$. In both reconstruction methods, the density effect on emission and ionization processes is included by using effective rate coefficients. They are evaluated from the collisional radiative model for a beam energy of $8 \mathrm{keV}$. The use of effective rate coefficients simplifies the density reconstruction procedure for complicated atomic processes. 


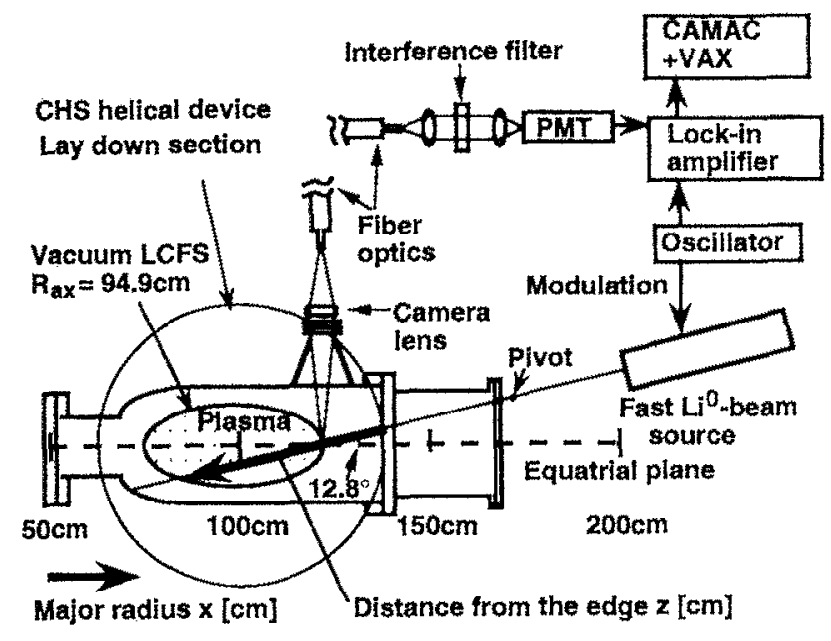

FIG. 1. Experimental setup for fast $\mathrm{Li}^{\mathrm{O}}$-beam probing on $\mathrm{CHS}$.

\section{EXPERIMENTAL SETUP}

An $8-\mathrm{keV} \mathrm{Li}^{0}$-beam probing system installed on the CHS is shown in Fig. 1. The CHS is a heliotron/torsatron device which has a major radius of $100 \mathrm{~cm}$ and a minor radius of $20 \mathrm{~cm}$. The pole number and the toroidal period number of the helical field coils are $l=2$ and $m=8$, respectively. A hydrogen plasma is produced by electron cyclotron resonance (ECR) using a $53-\mathrm{GHz}, 150 \mathrm{~kW}$ microwave source. The plasma is additionally heated by $45 \mathrm{keV}$, 1-MW neutral beam injection (NBI). The fast $\mathrm{Li}^{\mathrm{O}}$-beam injector consists of an ion gun with a thermoionic emission $\mathrm{Li}^{+}$source ( $\beta$-eucryptite), a Pierce extractor, a cylindrical lens, and a neutralizing cell containing $C s$ vapor. ${ }^{2,3}$ The equivalent neutral beam current density is $50 \mu \mathrm{A} / \mathrm{cm}^{2}$ with a diameter of $15-20 \mathrm{~mm}$ at FWHM. The Li I resonance line $(6708 \AA)$ is detected by a photomultiplier tube and an optical system consisting of lenses, fiber optics, and an interference filter with a FWHM of $15 \AA$. In order to improve the signal-to-noise ratio $(S / N)$, the beam is modulated at $10 \mathrm{kHz}$ and a lock-in amplifier is used. The $\mathrm{Li}$ I emission profile is obtained by changing the point of observation shot by shot. Since the beam injection line is tilted from the equatorial plane, we introduce two coordinates. The electron density is reconstructed along the $z$-coordinate which represents the distance from the wall along the beam line. The electron densities thus obtained are plotted as a function of major radius $x$. In the present setup, the observable region is $x \sim 113-126 \mathrm{~cm}$, which corresponds to the edge region in CHS.

\section{LITHIUM EMISSION AND IONIZATION PROCESSES}

$\mathrm{Li}^{0}$ atoms are excited or ionized by various collisional processes with plasma electrons, protons, and impurities. In addition, ionization and de-excitation from the lithiurnexcited state influences both emission and ionization processes. These effects should be taken into account when the target plasma has a density above $10^{12} \mathrm{~cm}^{-3}$. Here we introduce effective emission and ionization rate coefficients which are functions of plasma density and impurity con-

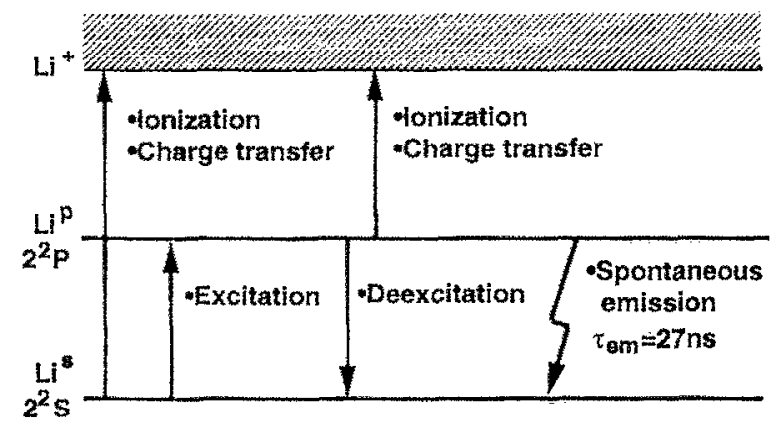

FIG. 2. Atomic processes of $\mathrm{Li}^{0}$ atoms in CHS edge plasma.

centration. The use of effective rate coefficients simplifies density reconstruction algorithms even though complicated atomic processes are included.

Electronic transition processes of $\mathrm{Li}^{0}$ atom are shown in Fig. 2. The rate coefficients for each process and their notations are summarized in Table I. These rate coeffcients are calculated from Refs. 13 and 14 or evaluated from scaling models ${ }^{15-17}$ for a beam energy of $8 \mathrm{keV}$ and a plasma temperature of $30 \mathrm{eV}$. The temperature dependence of the rate coefficients in the range of $10-100 \mathrm{eV}$ is small enough to be neglected. Electron impact processes are most important when evaluating both beam emission and attenuation. On the other hand, charge transfer processes with plasma protons and impurities are important when evaluating beam attenuation. In the case of an $8-\mathrm{keV} \mathrm{Li}^{\circ}$ beam, the rate coefficient for charge transfer to protons is about the same order as that for electron impact ionization. Multicharged impurity ions have a more serious influence through charge transfer. In the classical model, ${ }^{17}$ higher charged states have greater cross sections for charge transfer. The typical impurity species are oxygen ions in CHS. Since the electron temperature is $300 \mathrm{eV}$, it is reasonable to assume that helium-like oxygen ion $\mathrm{O}^{6+}$, with an ionization potential of $739 \mathrm{eV}$, is the typical and most harmful impurity for $\mathrm{Li}^{0}$-beam probing. In order to simplify the analysis, we introduce total rate coefficients which include

TABLE I. Rate coefficients for lithium atomic processes.

$$
\text { Rate coefficient }\langle o v\rangle\left[\mathrm{cm}^{3} / \mathrm{s}\right]
$$

Electron impact Proton impact $O^{6 /}$ impact

\begin{tabular}{|c|c|c|c|}
\hline $\begin{array}{l}\text { Excitation } \\
\mathrm{Li}^{*} \Rightarrow \mathrm{Li}^{p}\end{array}$ & $\begin{array}{l}\left\langle\sigma_{\mathrm{ex}} v_{e}\right\rangle \\
=8.2 \times 10^{-7}\end{array}$ & $\begin{array}{l}\left\langle\sigma_{\mathrm{ex}} v_{\mathrm{Li}-p}\right\rangle \\
=7.1 \times 10^{-8}\end{array}$ & neglected \\
\hline $\begin{array}{l}\text { Deexcitation } \\
\mathrm{Li}^{p} \Rightarrow \mathrm{Li}^{s}\end{array}$ & $\begin{array}{l}\left\langle\sigma_{\operatorname{dez}} v_{e}\right\rangle \\
=2.6 \times 10^{-7}\end{array}$ & $\begin{array}{l}\left\langle\sigma_{\mathrm{dex}}{ }^{2} h_{i-p}\right\rangle \\
=2.4 \times 10^{-8}\end{array}$ & neglected \\
\hline $\begin{array}{l}\text { Ionization } \\
\mathrm{Li} \mathrm{i}^{+} \Rightarrow \mathrm{Li}^{+}\end{array}$ & $\begin{array}{l}\left\langle\sigma_{\mathrm{is}} v_{e}\right\rangle \\
=8.0 \times 10^{-8}\end{array}$ & $\begin{array}{l}\left\langle\sigma_{i s} v_{L i-p}\right\rangle \\
=7.6 \times 10^{-9}\end{array}$ & neglected \\
\hline $\begin{array}{l}\text { Ionization } \\
\mathrm{Lip}^{p} \Rightarrow \mathrm{Li}^{+}\end{array}$ & $\begin{array}{l}\left\langle\sigma_{\mathrm{ip}} v_{\mathrm{e}}\right\rangle \\
=2.3 \times 10^{-7}\end{array}$ & $\begin{array}{l}\left\langle\sigma_{\mathrm{ip} p} \nu_{\mathrm{Li}-p}\right\rangle \\
=2.5 \times 10^{-8}\end{array}$ & neglected \\
\hline $\begin{array}{l}\text { Charge transfer } \\
\mathrm{Li}^{3} \Rightarrow \mathrm{Li}^{+}\end{array}$ & & $\begin{array}{l}\left\langle\sigma_{\mathrm{cs}} v_{\mathrm{Li}-p}\right\rangle \\
=9.4 \times 10^{-8}\end{array}$ & $\begin{array}{l}\left\langle\sigma_{\mathrm{cs}} v_{\mathrm{Li}-\mathrm{O}^{6}+}\right\rangle \\
=1.6 \times 10^{-6}\end{array}$ \\
\hline $\begin{array}{l}\text { Charge transfer } \\
\mathrm{Li}^{p} \Rightarrow \mathrm{Li}^{+}\end{array}$ & & $\begin{array}{l}\left\langle\sigma_{\mathrm{cp}} v_{\mathrm{Li}-\rho}\right\rangle \\
=2.1 \times 10^{-7}\end{array}$ & $\begin{array}{l}\left\langle\sigma_{\mathrm{cp}} v_{\mathrm{L}-0^{6}+}\right\rangle \\
=3.8 \times 10^{-6}\end{array}$ \\
\hline
\end{tabular}


electron, proton, and impurity impact processes. For example, a total rate coefficient for ionization from the ground state, $\left\langle\sigma_{\text {is }} v\right\rangle$, is expressed as follows:

$$
\begin{aligned}
n_{e}\left\langle\sigma_{\mathrm{is}} v\right\rangle n_{\mathrm{Li}^{s}}= & n_{e}\left\langle\sigma_{\mathrm{is}} v_{e}\right\rangle n_{\mathrm{Li}^{s}}+n_{p}\left(\left\langle\sigma_{\mathrm{is}} v_{\mathrm{Li}-p}\right\rangle+\left\langle\sigma_{\mathrm{cs}} v_{\mathrm{Li}-p}\right\rangle\right) n_{\mathrm{Li}^{s}} \\
& +n_{\mathrm{O}^{\sigma}+}\left\langle\sigma_{\mathrm{cs}} v_{\mathrm{Li}-\mathrm{O}^{6+}}\right\rangle n_{\mathrm{Li}^{s}} \\
= & n_{e}\left\{\left\langle\sigma_{\mathrm{is}} v_{e}\right\rangle+(1-6 \xi)\left(\left\langle\sigma_{\mathrm{is}} v_{\mathrm{Li}-p}\right\rangle\right.\right. \\
& \left.\left.+\left\langle\sigma_{\mathrm{cs}} v_{\mathrm{Li}-p}\right\rangle\right)+\xi\left\langle\sigma_{\mathrm{cs}} v_{\mathrm{Li}-\mathrm{O}^{6+}}\right\rangle\right\} n_{\mathrm{Lij}} s
\end{aligned}
$$

where $n_{e}, n_{p}, n_{\mathrm{O}^{6+}}$, and $n_{\mathrm{Li}}$ are the electron, proton, $\mathrm{O}^{6+}$ ion, and ground state $\mathrm{Li}^{0}$-atom densities, respectively. The impurity concentration ratio $\xi$ is defined as $\xi$ $=n_{\mathrm{O}^{6}+} / n_{\mathrm{e}}$. In a similar way total rate coefficients for ionization from the excited state $\left\langle\sigma_{\mathrm{ip}} v\right\rangle$, for excitation $\left\langle\sigma_{\mathrm{ex}} v\right\rangle$ and for deexcitation $\left\langle\sigma_{\mathrm{dex}} v\right\rangle$ are defined.

In the collisional radiative model, the rate equation for the excited state of the $\mathrm{Li}^{0}$ atom is expressed as follows:

$\frac{d n_{\mathrm{L} j p}}{d t}=n_{e}\left\langle\sigma_{\mathrm{ex}} v\right\rangle n_{\mathrm{Li}^{s}}-n_{e}\left\langle\sigma_{\mathrm{ip}} v\right\rangle n_{\mathrm{L} j p}-n_{e}\left\langle\sigma_{\mathrm{dex}} v\right\rangle n_{\mathrm{L} j^{p}}-\frac{n_{\mathrm{Li} p}}{\tau_{\mathrm{em}}}=0$,

where $n_{\mathrm{L}, p}$ is the $\mathrm{Li}^{0}$-atom density in the excited state and $\tau_{\mathrm{em}}$ is its lifetime (27 ns). The spatial resolution for the density measurement is expressed as $\tau_{\mathrm{em}} \times v_{\mathrm{Li}} \sim 1.3 \mathrm{~cm}$. Solving Eq. (2), $n_{\mathrm{L} j}$ is expressed as a function of total $\mathrm{Li}^{0}$-atom density $n_{\mathrm{Li}}=n_{\mathrm{Li} s}+n_{\mathrm{Lip}}$ and the electron density $n_{e}$.

$$
n_{\mathrm{L} j}=\frac{n_{e}\left\langle\sigma_{\mathrm{ex}} v\right\rangle \tau_{\mathrm{eax}}}{1+n_{e}\left(\left\langle\sigma_{\mathrm{ip}} v\right\rangle+\left\langle\sigma_{\mathrm{dex}} v\right\rangle+\left\langle\sigma_{\mathrm{ex}} v\right)\right) \tau_{\mathrm{em}}} n_{\mathrm{Li}} .
$$

Since the ratio of $n_{\mathrm{L} ; p}$ to $n_{\mathrm{Li}}$ depends on $n_{e}$, effective emission and ionization rate coefficients are functions of $n_{e}$. The effective emission rate coefficient $\left\langle\sigma_{\mathrm{em}} v\right\rangle_{\mathrm{eff}}$ for the $\mathrm{Li}$ I resonance line is defined as follows:

$$
n_{\mathrm{e}}\left\langle\sigma_{\mathrm{em}} v\right\rangle_{\mathrm{eff}} n_{\mathrm{Li}}=\frac{n_{\mathrm{L} j}}{\tau_{\mathrm{em}}} .
$$

The effective ionization rate coefficient $\left\langle\sigma_{i} \nu\right\rangle_{\text {eff }}$ is defined as follows:

$$
n_{e}\left\langle\sigma_{i} \nu\right\rangle_{\mathrm{eff}} n_{\mathrm{Li}}=n_{e}\left\langle\sigma_{\mathrm{is}} v\right\rangle n_{\mathrm{Li}}+n_{e}\left\langle\sigma_{\mathrm{ip}} v\right\rangle n_{\mathrm{Li} p} .
$$

The density dependence of $\left\langle\sigma_{\mathrm{em}} v\right\rangle_{\mathrm{eff}}$ and $\left\langle\sigma_{i} v\right\rangle_{\mathrm{eff}}$ is shown in Fig. 3. In low-density plasmas $\left(n_{e} \sim 10^{11} \mathrm{~cm}^{-3}\right)$, $\left\langle\sigma_{\mathrm{em}} v\right\rangle_{\mathrm{eff}}$ and $\left\langle\sigma_{p} \nu\right\rangle_{\mathrm{eff}}$ are nearly equal to $\left\langle\sigma_{\mathrm{ex}} v\right\rangle$ and $\left\langle\sigma_{\mathrm{is}} v\right\rangle$, respectively. In higher density plasmas $\left(n_{e}>10^{12} \mathrm{~cm}^{-3}\right)$, they start to deviate. At $n_{e}=10^{13} \mathrm{~cm}^{-3}$, for example, $\left\langle\sigma_{\mathrm{em}} v\right\rangle_{\mathrm{eff}}$ decreases by $31 \%$ and $\left\langle\sigma_{i} \nu\right\rangle_{\mathrm{eff}}$ increases by $43 \%$. This effect greatly influences the density measurement.

The impurity effect on these effective rate coefficients is calculated assuming $Z_{\text {eff }}=2$, which is typical in the CHS neutral beam heated plasmas. The results are shown in the same figure. It is shown that the increase of $\left\langle\sigma_{i} v\right\rangle_{\text {eff }}$ is about $20 \%$ for the $8-\mathrm{keV}$ beam. On the other hand, the decrease of $\left\langle\sigma_{\mathrm{em}} v\right\rangle_{\mathrm{eff}}$ is only $2 \%-3 \%$. The impurity effect is relatively small when the beam energy is low. It should be noted, however, that if the beam energy is increased to 20 $\mathrm{keV}$, the impurity effect becomes considerable. ${ }^{18}$

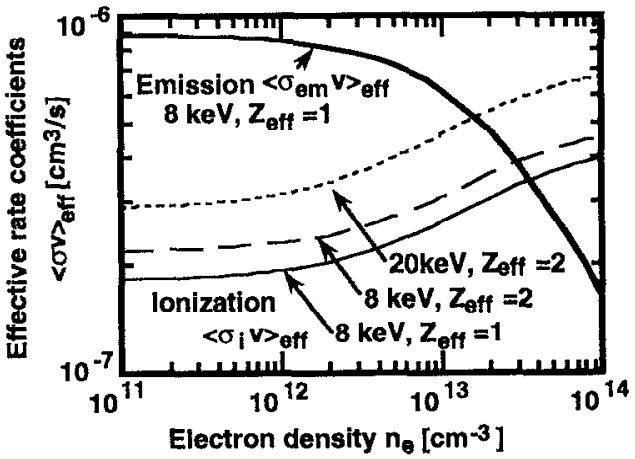

FIG. 3. Density dependence of the effective emission and ionization rate coefficients.

\section{METHOD I: ELECTRON DENSITY RECONSTRUCTION WITH GAS CALIBRATION}

Method I for electron density reconstruction is based on a simple emission method. First we will explain the principle of this method. The electron density $n_{e}(z)$ is obtained directly from the $\mathrm{Li}$ I emission signal, $I_{\mathrm{Li}}(z)$ [V], from the $\mathrm{Li}^{0}$-atoms injected into the plasma. The beam density and the sensitivity of the optical system are calibrated by injecting the $\mathrm{Li}^{0}$ beam into a target gas. Helium gas at a pressure of around $10^{-4}$ Torr is used for calibration. In this pressure range, beam attenuation can be neglected. The emission signal $I_{8}[\mathrm{~V}]$ from the $\mathrm{Li}^{0}$ atoms injected into calibration gas is written as Eq. (6) using the He gas density, $n_{g}\left[\mathrm{~cm}^{-3}\right]$, the excitation cross section for collision with $\mathrm{He}, \sigma_{\mathrm{ex}-\mathrm{g}}\left[\mathrm{cm}^{2}\right]$, and the beam velocity $v_{\mathrm{Li}}$ $[\mathrm{cm} / \mathrm{s}]$.

$$
I_{g}=n_{g} \sigma_{\mathrm{ex}-g} v_{\mathrm{Li}} n_{\mathrm{Li}}(0) \frac{\Omega}{4 \pi} V \eta
$$

where $\Omega$ and $V$ are the solid angle [str] and observation volume $\left[\mathrm{cm}^{3}\right]$ of the optical detection system, and $\eta[\mathrm{V} \cdot \mathrm{s}]$ is the sensitivity of the optical detector including the gain of the amplifier. On the other hand, the signal output $I_{\mathrm{Li}}(z)[\mathrm{V}]$ from $\mathrm{Li}^{0}$ atoms injected into the plasma is written as

$$
I_{\mathrm{Li}}(z)=n_{e}(z)\left\langle\sigma_{\mathrm{em}} v\right\rangle_{\mathrm{eff}} n_{\mathrm{Li}}(z) \frac{\Omega}{4 \pi} V \eta
$$

where $n_{e}(z)$ is the electron density. From Eqs. (6) and (7), $n_{e}(z)$ can be obtained as follows:

$$
n_{e}(z)=\frac{I_{\mathrm{Li}}(z)}{I_{g}} \frac{\sigma_{\mathrm{ex}}-v_{\mathrm{Li}}}{\left\langle\sigma_{\mathrm{em}} v\right\rangle_{\mathrm{eff}}} n_{g} .
$$

The edge $n_{e}$ profiles for ECR sustained plasmas have been measured by this simple emission method. A typical example for this measurement is shown in Fig. 4. The impurity effect is neglected since $\left\langle\sigma_{\mathrm{em}} v\right\rangle_{\text {eff }}$ has a small $Z_{\text {eff }}$ dependence. The data from Thomson scattering on the same magnetic flux surface but separated by $67.5^{\circ}$ in the 


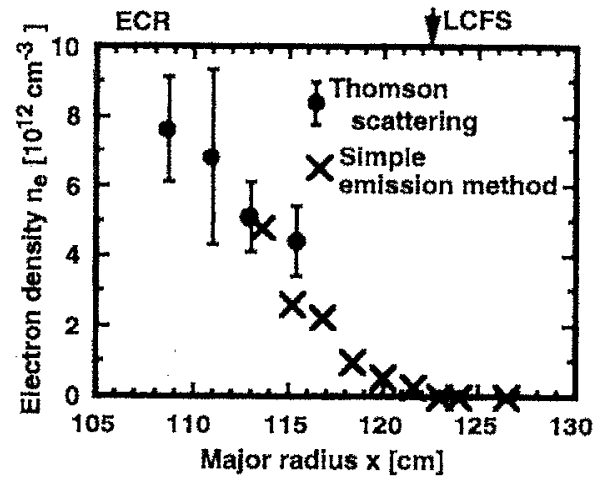

FIG. 4. Electron densities measured by the simple emission method in the ECR sustained plasma. Thomson scattering data taken at a different toroidal position are shown for comparison.

toroidal direction is shown. This figure demonstrates that the two $n_{e}$ profiles are smoothly connected. This method is very simple and straightforward, but the application is limited to low-density plasmas in which beam attenuation is negligible.

If we evaluate the lithium atom density taking attenuation into account, we can apply this method to higher density plasmas. In method $\mathrm{I}$, the $n_{e}$ profile is reconstructed using the results from the simple emission method as an initial condition for calculation. Beam attenuation due to ionization is expressed as

$$
\frac{1}{n_{\mathrm{Li}}(z)} \frac{d n_{\mathrm{Li}}(z)}{d z}=-\frac{n_{e}(z)\left\langle\sigma_{f} v\right\rangle_{\mathrm{efr}}}{v_{\mathrm{Li}}} .
$$

Li I emission signal can be recalculated numerically from the $n_{e}$ profile using Eqs. (6), (7), and (9). The recalculated emission signal $\Gamma_{\mathrm{Li}}^{*}(z)$ is expressed as

$$
I_{\mathrm{Li}}^{*}(z)=I_{g} \frac{n_{e}(z)\left\langle\sigma_{\mathrm{em}} v\right\rangle_{\mathrm{eff}}}{n_{g} \sigma_{\mathrm{ex}-g} v_{\mathrm{Li}}} \exp \left[-\int_{0}^{z} \frac{\left\langle\sigma_{i} v\right\rangle_{\mathrm{eff}}}{v_{\mathrm{Li}}} n_{g}(\zeta) d \xi\right]
$$

It should be noted that $\left\langle\sigma_{e m} v\right\rangle_{\text {eff }}$ and $\left\langle\sigma_{i} \nu\right\rangle_{\text {eff }}$ in Eq. (10) are functions of $n_{e}(z)$. The $n_{e}$ profile is determined by iteration so that the observed emission signal $I_{\mathrm{Li}}(z)$ coincides with the numerically calculated $I_{\mathrm{Li}}^{*}(z)$. Hence the $n_{e}$ profile is calculated from the plasma edge toward the center. The result from the simple emission method putting $\left\langle\sigma_{\mathrm{cm}} v\right\rangle_{\mathrm{eff}}=\left\langle\sigma_{\mathrm{ex}} v\right\rangle$ is used as an initial value for iteration.

An example of the $n_{e}$ profile for a typical discharge with NBI heating is shown in Fig. 5. The magnetic axis is set at the major radius $x=94.9 \mathrm{~cm}$ and the position of the last closed flux surface (LCFS) is at $x=122.5 \mathrm{~cm}$. The $I_{\mathrm{Li}}$ profile is obtained by spline interpolation from the discrete data, which is shown with the data points. It is obvious that the beam attenuation and the decrease of $\left\langle\sigma_{\mathrm{em}} v\right\rangle_{\mathrm{eff}}$ greatly influences the reconstructed density. The increase of $\langle\sigma i v\rangle_{\text {eff }}$ due to the impurity effect makes the obtained $n_{e}$ greater. The impurity effect, however, is small for a beam energy of $8 \mathrm{keV}$ as discussed before.

The applicable spatial range of method $I$ is limited to the region from the plasma edge to the position where the

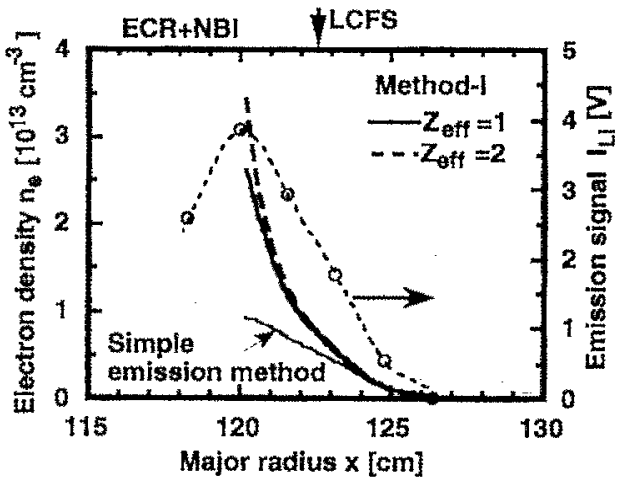

FIG. 5. Results of electron density reconstruction by method I in the NBI heated plasma. Initial electron density profile for the iteration is obtained by the simple emission method.

emission signal has its peak $\left(z<z_{\text {peak }}\right)$. This is because only a several percent experimental error or misevaluation of the atomic data makes the reconstructed $n_{e}$ profile diverge. It should be noted that the accuracy of calibration greatly infuences the reconstructed density.

\section{METHOD II: ELECTRON DENSITY RECONSTRUCTION FROM THE SHAPE OF EMISSION PROFILE}

The second method, classified as method II, reconstructs the $n_{e}$ profile from the shape of the emission signal and the atomic data. In this method, $n_{\mathrm{Li}}(z)$ is determined by integrating the $\mathrm{Li}$ I emission intensity from position $z$ toward the emission tail-off position $z_{1}$. In other words, the beam density at position $z$ is measured from the emission profile. By eliminating $n_{e}(z)$ from Eqs. (7) and (9), the local beam density $n_{\mathrm{Li}}(z)$ is expressed as

$$
n_{\mathrm{Li}}(z)=\int_{z}^{z_{1}} \frac{\left\langle\sigma_{i} v\right\rangle_{\mathrm{eff}}}{\left\langle\sigma_{\mathrm{em}} v\right\rangle_{\mathrm{eff}}} I_{\mathrm{Li}}(\zeta) d \zeta / v_{\mathrm{Li}} V \frac{\Omega}{4 \pi} \eta
$$

$z_{1}$ is set at the position where the $\mathrm{S} / \mathrm{N}$ of the emission signal becomes less than unity. This method is applicable only when position $z_{1}$ is within the observation window. From Eqs. (7) and (11), the parameters for the optical system, such as $\Omega, V$, and $\eta$, are canceled out and $n_{e}(z)$ is expressed as

$$
n_{e}(z)=I_{\mathrm{Li}}(z) v_{\mathrm{Li}} /\left\langle\sigma_{\mathrm{em}} v\right\rangle_{\mathrm{eff}} \int_{z}^{z_{1}} \frac{\left\langle\sigma_{i} v\right\rangle_{\mathrm{eff}}}{\left\langle\sigma_{\mathrm{em}} v\right\rangle_{\mathrm{eff}}} I_{\mathrm{Li}}(\zeta) d \zeta
$$

A great advantage of this method is that there is no need for calibration of the optical system or the beam density. Since $\left\langle\sigma_{\mathrm{erm}} v\right\rangle_{\mathrm{eff}}$ and $\left\langle\sigma_{i} v\right\rangle_{\mathrm{eff}}$ are functions of $n_{e}(z)$, the $n_{e}$ profile is calculated by iteration. The initial $n_{e}$ profile for iteration is approximated by neglecting the density effect by putting $\left\langle\sigma_{\mathrm{em}} v\right\rangle_{\mathrm{eff}}=\left\langle\sigma_{\mathrm{ex}} v\right\rangle$ and $\left\langle\sigma_{i} v\right\rangle_{\mathrm{eff}}=\left\langle\sigma_{\mathrm{is}} v\right\rangle$. It must be noted that the accuracy of the reconstructed density strongly depends on the reliability of $\left\langle\sigma_{i} v\right\rangle_{\mathrm{eff}}$.

Examples of $n_{e}$ profiles reconstructed by method II are shown in Fig. 6, where the same emission profile in Fig. 5 


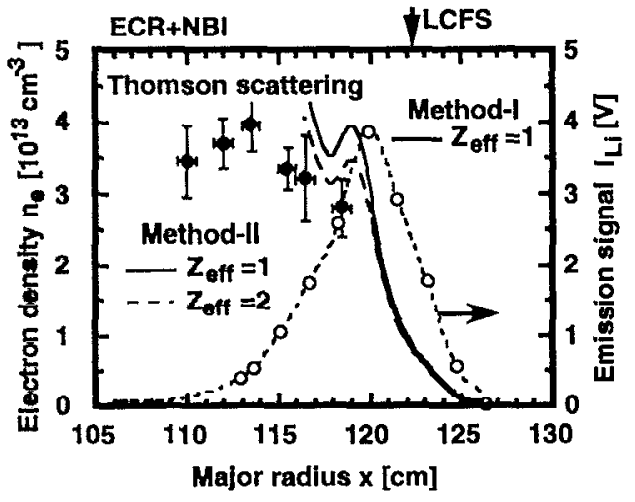

FIG. 6. Results of electron density reconstruction by method II in the NBI heated plasma. For comparison, the results obtained by method I and Thomson scattering data on the same magnetic flux surface are shown.

is used. Ten time iteration is carried out to obtain this $n_{e}$ profile. The $I_{\mathrm{Li}}$ data inside the observation limit $(x \leqslant 113$ $\mathrm{cm}$ ) is extrapolated by an exponential curve. Position $z_{1}$ is determined from this curve. The $n_{e}$ profile from method I is also shown for comparison. The $n_{e}$ profiles obtained by methods I and II show good agreement although these methods determine $n_{\mathrm{Li}}(z)$ in quite different ways. The impurity effect on method II is also small. The increase of $\left\langle\sigma_{i} \nu\right\rangle_{\text {eff }}$ due to the impurity effect, however, makes the reconstructed $n_{e}$ value smaller.

The accuracy of the reconstructed density becomes worse as $z$ becomes larger since this method includes the integration from the emission tail-off where the accuracy of measurement is not good. From our calculation, the reliable range where the error is within $20 \%$ is from the plasma edge to the position where the $I_{\mathrm{Li}}$ is attenuated to the $70 \%$ level of its peak $\left(z<z_{70 \% \text { peak }}\right)$. The small hump just inside the emission peak is not considered to be real. It occurs because the spline curve fitting is unsatisfactory due to the rough data points. The $n_{e}$ profile is compared with that from ruby laser Thomson scattering on the same magnetic flux surface but at a different toroidal position. A finite beta shift of the plasma column is taken into account for the conversion of the two coordinates. ${ }^{19}$ The two profiles are consistent, although stricter point-to-point correspondence is necessary for a detailed comparison. The coincidence of the electron densities measured in three different ways demonstrates the reliability of the diagnostic system and the density reconstruction methods.

\section{SELECTION OF THE ELECTRON DENSITY RECONSTRUCTION METHOD}

The two $n_{e}$ profile reconstruction methods have different applicable depths and average densities. By selecting methods I or II, we can obtain $n_{e}$ profiles in various kinds of discharges from low-density $\left(10^{11} \mathrm{~cm}^{-3}\right)$ to highdensity $\left(10^{14} \mathrm{~cm}^{-3}\right)$ plasmas. The temporal evolution of the $n_{e}$ profile in identical discharges, as shown in Fig. 7, is obtained by selecting the appropriate reconstruction method. The plasma is produced by ECR and additionally

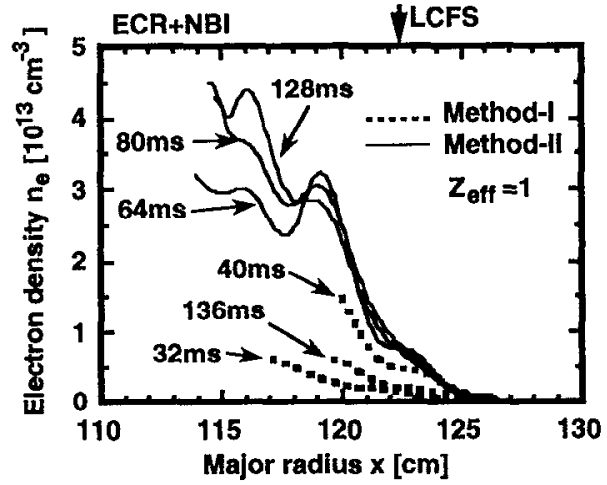

FIG. 7. Temporal evolution of electron density profile in the CHS discharge. The plasma is produced by ECR and additionally heated by NBI from 28 to $128 \mathrm{~ms}$.

heated by NBI from 28 to $128 \mathrm{~ms}$ in the discharge sequence. The $n_{e}$ profile at $80 \mathrm{~ms}$ is the same as that shown in Figs. 5 and 6. Method II is used when the emission signal is strongly attenuated within the observation window $(z \geqslant 113 \mathrm{~cm})$, since the applicable depth of method II is larger than that of method I. On the other hand, method I is used when the beam is weakly attenuated. It can be seen from the figure that the plasma density gradually increases during NBI injection until it reaches a steady state and then decreases afterward.

The applicable depth $\left(z_{\mathrm{Lim}}\right)$ and the applicable average density $\left(\overline{n_{e}}\right)$ for each $n_{e}$ reconstruction procedure is estimated for the $\mathrm{Li}^{0}$-beam probe system on CHS where the observation coverage is $15 \mathrm{~cm} . z_{\operatorname{Lim}}$ is the deepest point at which the accuracy of the reconstruction is sufficient. $z_{\mathrm{Lim}}$ for each method is shown as a function of $\bar{n}_{e}$ in Fig. 8. For simplicity, $n_{e}$ is assumed to increase linearly with respect to $z$. Criteria in determining applicable $z_{\mathrm{Lim}}$ and $\overline{n_{e}}$ are summarized in Table II. It can be seen that method I covers a wide range of electron densities. On the other hand, method II has the advantage of a larger applicable depth. The lower limit of the applicable average density for method II can be reduced by extending the observation coverage so that the emission tail-off is within the view of the optical system.

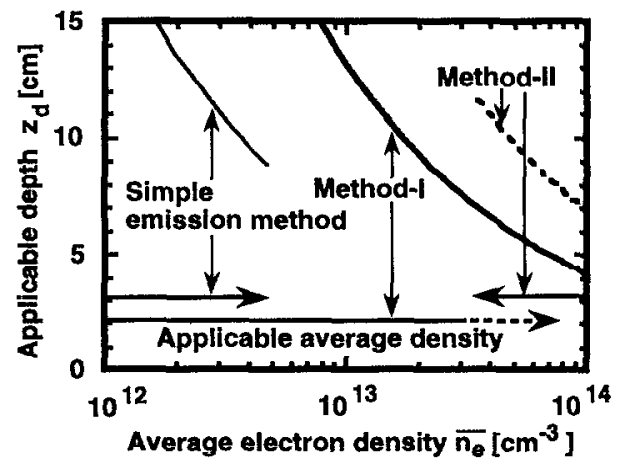

FIG. 8. The applicable depth $\left(z_{d}\right)$ and the applicable average density $\left(\overline{n_{e}}\right)$ for the simple emission method, methods I and II. 
TABLE II. Criteria for determining the applicable depth $\left(z_{d}\right)$ and the applicable average density $\left(\bar{n}_{e}\right)$ for the simple emission method, methods I and II.

\begin{tabular}{|c|c|c|c|}
\hline & Simple emission method & Method I & Method II \\
\hline$z_{d}$ & $\frac{n_{\mathrm{Li}}\left(z_{d}\right)}{n_{\mathrm{Li}}(0)}=0.9$ & $z_{d}=z_{\text {peak }}$ & $z_{d}=z_{70 \% \text { peak }}$ \\
\hline$\overline{n_{e}}$ & $\frac{\left\langle\sigma_{\mathrm{em}} v\right\rangle_{\text {eff }}}{\left\langle\sigma_{\mathrm{ex}} v\right\rangle}>0.95$ & $z_{d}>5 \mathrm{~cm}$ & $\frac{n_{\mathrm{Li}}(15 \mathrm{~cm})}{n_{\mathrm{Li}}(0)}<0.1$ \\
\hline
\end{tabular}

\section{ACKNOWLEDGMENTS}

One of the authors (S.S.) would like to thank Dr. K. N. Sato, Dr. T. Kato, Dr. M. Fukao, and Dr. Y. Uesugi for valuable discussions. Another (M.U.) would like to thank the partial support of JSPS (Japan) and CNPq (Brazil). Helpful discussions with Dr. K. MeCormick, a visiting professor at NIFS, are also acknowledged. We also thank Dr. K. Matsuoka, Dr. S. Okamura, Dr. K. Nishimura, Dr. H. Yamada, and other CHS staff for continuous help in the experiments. This work was supported in part by a Grantin-Aid of Scientific Research from Japan Ministry of Education, Science and Culture (JSPS Fellowship, No. 3293).

${ }^{1}$ K. Kadota, K. Tsuchida, Y. Kawasumi, and J. Fujita, Plasma Phys. 20, 1011 (1978).

${ }^{2}$ H. Iguchi, K. Kadota, K. Takagi, T. Shoji, M. Hosokawa, M. Fujiwara, and $\mathrm{K}$. Ikegami, Rev, Sci. Instrum. 56, 1056 (1985).
${ }^{3}$ A. Pospieszczyk, F Aumayr, H. L. Bay, E. Hintz, P. Leismann, Y. T. Lie, G. G. Ross, D. Rusbuild, R. P. Schorn, B. Schweer, and H. Winter, J. Nucl. Mater. 128\&129, 574 (1989).

${ }^{4} \mathrm{~K}$. McCormick and ASDEX team, Rev. Sci. Instrum. 56, 1063 (1985).

${ }^{\prime} M$. Ueda, H. Iguchi, S. Sasaki, J. Fujita, and CHS group, J. Nucl. Mater. 196-198, 923 (1992).

${ }^{6}$ R. C Isler, L. E. Murray, S. Kasai, J. L. Dunlap, S. C. Bates, P. H. Edmonds, E. A. Lazarus, C. H. Ma, and M. Murakami, Phys. Rev. A 24, 2701 (1981).

${ }^{\top}$ L. Weirelbaum, E. Hayess, U. Wenzel, J, Badalec, K. Jakubka, L. Kryska., J. Stoeckel, M. Valovic, and F. Zacek, J. Nucl. Mater. $1768177,904(1990)$.

${ }^{8}$ A. Komori, A. Yonesu, S. Nagai, T. Mizunchi, M. Harada, H. Matsuura, F. Sano, H. Zushi, S. Sudo, M. Nakasuga, Y. Kawai, and T. Obiki, Jpn. J. A.ppl. Phys. 30, 3526 (1991).

${ }^{9}$ A. Pospieszczyk and G. G. Ross, Rev. Sci. Instrum. 59, 605 (1988).

${ }^{10} \mathrm{~S}$. Sasaki, S. Takamura, and K. Kadota, Kakuyugo Kenkyu (in Japanese) 62,282 (1989).

${ }^{11}$ S. Sasaki, S. Takamura, Y. Uesugi, Y. Ohkouchi, and K. Kadota, Rev. Sci. Instrum. (to be published).

${ }^{12}$ D. Michaud, G. G. Ross, E. Hadded, H. H. Mai, A. Pospieszczyk, and J. P. St-Germain, Rev. Sci. Instrum. 63, 5698 (1992).

${ }^{13}$ F. Aumayr and H. Winter, Atomic Data Base of Lithium-Beam Activated Plasma Diagnasties, Institute fir Allgemeine Physik Technishe Universität, Vienna. IAP-Report 1/87 (1987).

${ }^{14}$ K. Kadota, A. Pospieszczyk, P. Bogen, and E. Hintz, IEEE Trans. Plasma Sci. PS-12, 262 (1984).

${ }^{15}$ W. Lotz, Astrophys. J. Suppl. Ser. 14, 207 (1967).

${ }^{16}$ R. E. Olson, J. Phys. B 13, 483 (1980).

${ }^{17}$ H. Ryufuku, K. Sasaki, and T. Watanabe, Phys. Rev. A 21, 745 (1980).

${ }^{18}$ J. Schweinzer, K. McCormick, S. Fielder, F. Aumyr, M. Pöckl, and H. Winter, in Proc. of 1992 Int. Conf. on Plasma Physics, Innsbrück (EPS, 1992), Part II, pp. 1163-1166.

${ }^{19}$ H. Yamada, K. Ida, H. Iguchi et al., Nucl. Fusion 32, 25 (1992). 